\title{
Stereo Matching with Non-Linear Diffusion
}

\author{
Daniel Scharstein* \\ Department of Computer Science \\ 5151 Upson Hall \\ Cornell University \\ Ithaca, NY 14853-7501 \\ schar@cs.cornell.edu
}

\author{
Richard Szeliski
}

\begin{abstract}
One of the central problems in stereo matching (and other image registration tasks) is the selection of optimal window sizes for comparing image regions. This paper addresses this problem with some novel algorithms based on iteratively diffusing support at different disparity hypotheses, and locally controlling the amount of diffusion based on the current quality of the disparity estimate. It also develops a novel Bayesian estimation technique which significantly outperforms techniques based on area-based matching (SSD) and regular diffusion. We provide experimental results on both synthetic and real stereo image pairs.
\end{abstract}

A short version of this paper appears in IEEE Computer Society Conference on Computer Vision and Pattern Recognition (CVPR'96), San Francisco, June 1996.

\footnotetext{
${ }^{*}$ Supported by funds of the National Science Foundation IRI-9057928.
} 


\section{Introduction}

Stereo correspondence is the problem of finding matching points in two or more images of the same scene, usually assuming known camera geometries. Two image points $p$ and $p^{\prime}$ match if they result from the projection of the same point $P$ in the scene, a property that is often approximated by a similarity constraint requiring, for example, $p$ and $p^{\prime}$ to have similar intensity or color. The desired output of a stereo correspondence algorithm is a disparity map, specifying the relative displacement of matching points between images.

The stereo correspondence problem is inherently underconstrained and further complicated by the fact that the images typically contain noise. Traditional approaches thus either try to only recover a subset of matches, or make additional assumptions. Feature-based approaches, belonging to the former category, only match points with a certain amount of local information (such as intensity edges), with the disadvantage of yielding only sparse disparity maps. In this paper we will focus on area-based approaches, which yield a dense disparity map by matching small image patches as a whole, relying on the assumption that nearby points usually have similar displacements.

A typical area-based stereo matching algorithm proceeds the following way: For each location in one image, find the displacement that aligns this location with the best matching location in the other image. The quality of a match is measured by comparing windows centered at the two locations, for example, using the sum of squared intensity differences (SSD).

A more general way of characterizing area-based algorithms is the following:

1. For each disparity under consideration, compute a per-pixel matching cost (e.g., squared intensity difference)

2. Aggregate support spatially (e.g. by summing over a window, or by diffusion)

3. Across all disparities, find the best match based on the aggregated support

4. Compute a sub-pixel disparity estimate (optional)

A central problem is to find the optimal size of the support region [Okutomi and Kanade, 1992; Kanade and Okutomi, 1994]. If the region is too small, a wrong match might be found due to ambiguities and noise. If the region is too big, it can no longer be matched as a whole due to foreshortening and occlusion, with the result of lost detail and blurring (or dislocating) object boundaries in the resulting disparity map.

In this paper, we first review the relevant literature and the basic idea of aggregating support (Sections 2 and 3). We then present some new algorithms that determine the best support region by iteratively diffusing support in a non-linear fashion (Section 4). We also develop a Bayesian model using explicit disparity distributions, and a novel iterative support aggregation algorithm based on this model (Section 5). We present comparative results for our algorithms in Section 6, and close with a discussion of future work. 


\section{Previous work}

In our discussion of related work we will focus on the different processing stages of the area-based algorithm outlined above. A general review of the stereo vision literature is beyond the scope of this paper. For surveys of the field see [Barnard and Fischler, 1982; Dhond and Aggarwal, 1989].

\subsection{Matching cost}

At the base of any matching algorithm is a matching cost that measures the (dis-)similarity of two locations. Matching costs can be defined locally (at pixel level), or over a certain area of support. Examples for local costs are absolute intensity differences [Kanade, 1994], squared intensity differences [Matthies et al., 1989], binary pixel matches [Marr and Poggio, 1976], edges [Baker, 1980], filtered images [Marr and Poggio, 1979; Jenkin et al., 1991; Jones and Malik, 1992], and measures based on gradient direction [Seitz, 1989] or gradient vectors [Scharstein, 1994]. Matching costs that are defined over a certain area of support include correlation [Ryan et al., 1980] and non-parametric measures [Zabih and Woodfill, 1994]. These can be viewed as a combination of the matching cost and aggregation stages.

\subsection{Evidence aggregation}

Aggregating support is necessary for stable matching. A support region can either be twodimensional at a fixed disparity (favoring fronto-parallel surfaces), or three-dimensional in $x-y$ - $d$ space (supporting slanted surfaces). Two-dimensional evidence aggregation has been done using square windows (traditional), Gaussian convolution [Scharstein, 1994], multiple windows anchored at different points [Intille and Bobick, 1994], and windows with adaptive sizes [Arnold, 1983; Okutomi and Kanade, 1992; Kanade and Okutomi, 1994]. Threedimensional support functions that have been proposed include limited disparity difference [Grimson, 1985], limited disparity gradient [Pollard et al., 1985], and Prazdny's coherence principle [Prazdny, 1985], which can be implemented using two diffusion processes [Szeliski and Hinton, 1985].

As mentioned above, some techniques, such as correlation and rank statistics which are defined over a fixed support region, can combine the cost and aggregation steps into one. Measures that can be accumulated in a separate step have the following advantages:

- efficiency: the measure can be aggregated with a single convolution (or box-filter) operation [Kanade, 1994],

- parallelizability: the aggregation step can be implemented by local iterative diffusion, making the algorithm suited for highly parallel architectures [Szeliski and Hinton, $1985]$, 
- adaptability: the measure can be aggregated over locally different support regions using either adjustable size windows [Kanade and Okutomi, 1994] or a non-uniform diffusion process (this paper).

\subsection{Disparity selection}

The easiest way of choosing the best disparity is to select at each pixel the minimum aggregated cost across all disparities under consideration ("winner-take-all"). A problem with this is that uniqueness of matches is only enforced for one image (the reference image), while points in the other image might get matched to multiple points. Cooperative algorithms employing symmetric uniqueness constraints are one attempt to solve this problem [Marr and Poggio, 1976]. Using dynamic programming techniques [Arnold, 1983; Ohta and Kanade, 1985; Cox, 1994; Intille and Bobick, 1994] is another way of selecting unique and consistent disparities. However, these techniques require the strict enforcement of ordering constraints [Yuille and Poggio, 1984].

\subsection{Sub-pixel disparity computation}

Sub-pixel disparity estimates can be computed by fitting a curve to the matching costs at the discrete disparity levels [Lucas and Kanade, 1981; Tian and Huhns, 1986; Matthies et al., 1989; Kanade and Okutomi, 1994]. This provides an easy way to increase the resolution of a stereo algorithm with little additional computation. However, to work well, the intensities being matched must vary smoothly.

\subsection{Other techniques}

Other stereo techniques include hybrid and iterative techniques, such as stochastic search [Szeliski and Hinton, 1985; Marroquin et al., 1987; Barnard, 1989] and joint matching and surface reconstruction [Hoff and Ahuja, 1989; Olsen, 1990]. Hierarchical (coarse-to-fine) matching is another important technique which allows for a larger range of disparities to be matched without excessive search [Quam, 1984; Witkin et al., 1987].

More than two images are used in multiframe stereo to increase stability of the algorithm [Bolles et al., 1987; Matthies et al., 1989; Kang et al., 1995]. A special case that easily fits in our framework is multiple baseline stereo, where all images have identical epipolar lines [Okutomi and Kanade, 1993]. In this case, the similarity measures between the reference image and all other images can be combined by summation into a single measure before the aggregation step.

Finally, occlusion is an important issue. Many approaches ignore the effects of occlusion; others try to minimize them by using a cyclopean disparity representation [Barnard, 1989], or try to recover occluded regions after the matching by cross-checking. Several authors have developed methods for dealing with occlusion explicitly, using Bayesian models and dynamic 
programming [Belhumeur and Mumford, 1992; Cox, 1994; Geiger et al., 1992; Intille and Bobick, 1994].

\subsection{Focus of this paper}

From the discussion above, it appears that most area-based stereo correspondence algorithms are composed of four tasks: computing a local matching cost; aggregating support spatially; finding the best disparity; and computing a sub-pixel disparity estimate. This framework allows us to compare different approaches that have been taken for each task in isolation, without being distracted by how the other tasks are being solved.

In this paper, we focus mainly on the second task: Aggregating support. We discuss various kinds of local diffusion, including a membrane model and a full distribution model, and contrast it to existing approaches, such as SSD and adaptive windows.

The other three task, although important, are not the central issue of this work. Unless noted otherwise, we use squared intensity differences as a matching cost, and, after the aggregation step, simply select the best disparity locally at each pixel. In the cases where we compute sub-pixel disparity estimates, we fit a parabola to the three cost values centered around the best disparity. It is important to keep in mind that the algorithms presented in this paper are independent of these choices and apply also to more sophisticated matching costs and disparity selection strategies.

\section{Aggregating support and the SSD algorithm}

In this section, we introduce the concept of disparity space, review the sum-of-squareddifferences (SSD) algorithm, and discuss the need for spatially-adaptive support regions.

\subsection{Disparity space}

Support for a match is defined over a three-dimensional disparity space $E(x, y, d)$. Formally, we define the initial (not yet aggregated) disparity space $E_{0}$ as

$$
E_{0}(x, y, d)=\rho\left(I_{L}(x+d, y)-I_{R}(x, y)\right),
$$

where $I_{L}, I_{R}$, are the intensity functions of the left and right image respectively, and $\rho$ measures the similarity between the two intensities, e.g., $\rho(l-r)=(l-r)^{2}$. This formulation uses $I_{R}$ as the reference image. After aggregating support into a final space $E(x, y, d)$, we can compute a disparity function

$$
d(x, y)=\arg \min _{d \in D} E(x, y, d)
$$

that represents the matches as offsets to the points in the right image. In practice, we will compute a discrete disparity field

$$
d_{i, j}=d\left(x_{i}, y_{j}\right)
$$



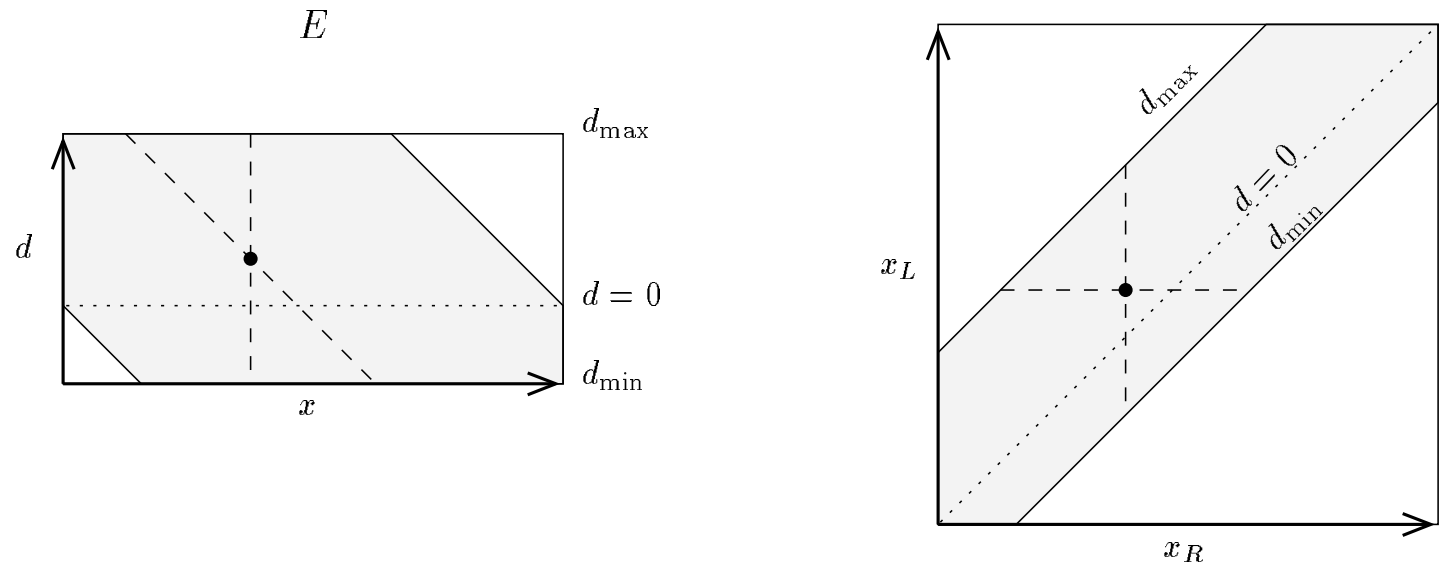

Figure 1: Slices through (skewed) disparity space $E$ and symmetric disparity space $\hat{E}$ for a fixed $y$. The lines of sight are shown as dashed lines for a given point in disparity space. The vertical dashed line corresponds to the right line of sight in both representations.

$E$ is a skewed version of the symmetric disparity space $\hat{E}$ [Marr and Poggio, 1976],

$$
\hat{E}\left(x_{R}, x_{L}, y\right)=\rho\left(I_{R}\left(x_{R}, y\right)-I_{L}\left(x_{L}, y\right)\right),
$$

which reflects that the matching problem is not biased towards either eye. In a symmetric setting, however, it is more difficult to enforce uniqueness for each pixel and to define the final disparity map (see Section 7 for a discussion). Figure 1 illustrates the shape of a slices through $E$ and $\hat{E}$ for a given $y$ and a limited disparity range $D=\left[d_{\min }, d_{\max }\right]$.

\subsection{SSD}

The standard sum-of-squared-differences algorithm (SSD) uses square windows to aggregate the evidence at each disparity. As mentioned before, choosing the right window size involves a trade-off between a noisy disparity map and blurring of depth boundaries. We will illustrate this using two synthetic image pairs. Both pairs have the same disparity pattern (see Figure 2): a central square floating in front of a background with constant disparity. Figure 2 (c) includes the occlusion information: the area displayed in white cannot be matched due to occlusion, and thus algorithms will assign arbitrary disparities in this region.

Figure 3 shows the two synthetic image pairs based on this disparity pattern. The first pair, ramp, is similar to the image pair in Fig. 5 in [Kanade and Okutomi, 1994] and is based on a linear intensity ramp in the direction of the baseline. Gaussian noise has been added to each image independently. The second image pair, $r d s$, is based on a binary random dot pattern using two gray levels with equal probability. No noise has been added to this image pair.

The two image pairs are quite different. The ramp pair has no local texture variation and constant gradients everywhere, except for the boundaries of the central square. The two 


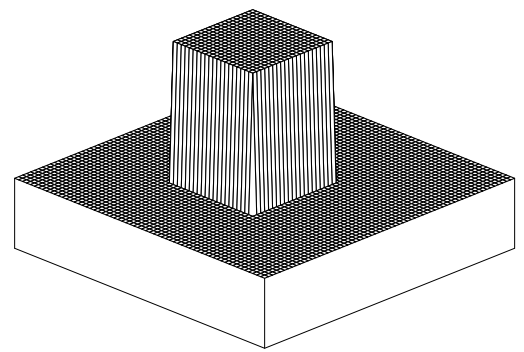

(a)

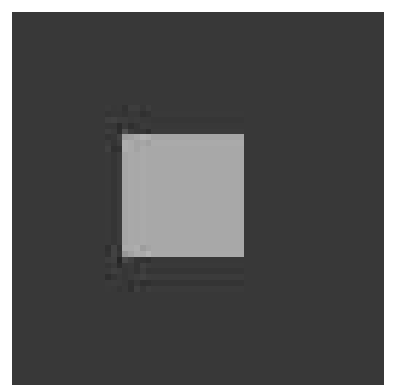

(b)

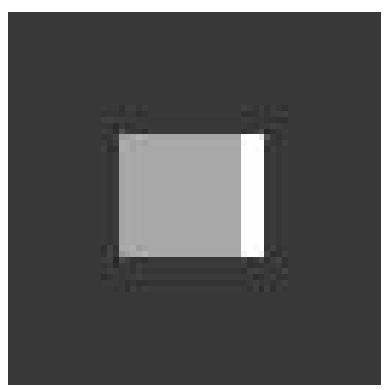

(c)

Figure 2: The disparity pattern for the ramp and $r d s$ pairs: (a) isometric plot; (b) gray-level encoding; (c) gray-level encoding with occlusion information.

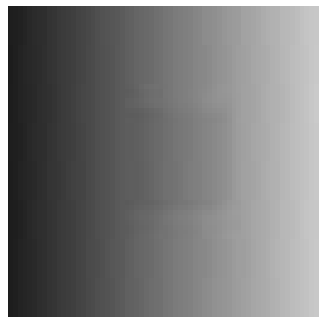

$\mathrm{L}$

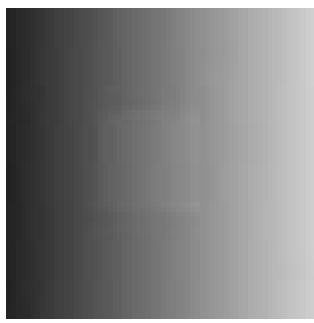

$\mathrm{R}$

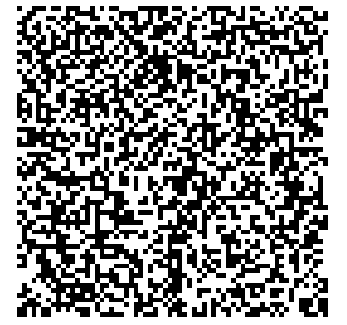

$\mathrm{L}$

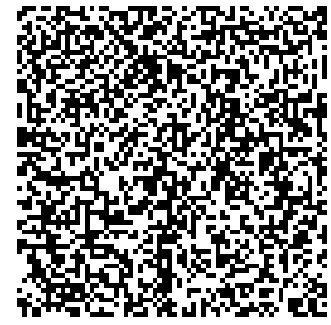

$\mathrm{R}$

Figure 3: Synthetic stereo pairs ramp (left) and rds (right).

images can only be matched by comparing absolute intensities, and any algorithm based on band-pass filtered intensities or gradients will fail (as will the human visual system). The $r d s$ pair, on the other hand, has strong local texture variation, but is highly ambiguous since pixels not in correspondence still have a $50 \%$ chance of matching.

Figure 4 shows the performance of the simple SSD algorithm on these two image pairs using two different window sizes, $w=3$ and $w=7$. As can be seen, the bigger window size yields a disparity map with less noise, but results in an overall blurring of the features (the "bumpiness" in the recovered disparities is due to sub-pixel disparity estimation). The effect on the two image pairs is quite different: in the ramp pair, the disparities are smoothed across the boundaries, while in the rds pair only the outlines of the square are blurred, i.e., the corners are rounded, while the two disparity levels of foreground and background are clearly recovered.

The latter effect, smoothing of object boundaries, is more common in real images pairs than the smoothing of disparities. The smoothing of disparities we observed in the ramp pair is a direct result of the ramp intensity pattern and the small local variations in intensity. 

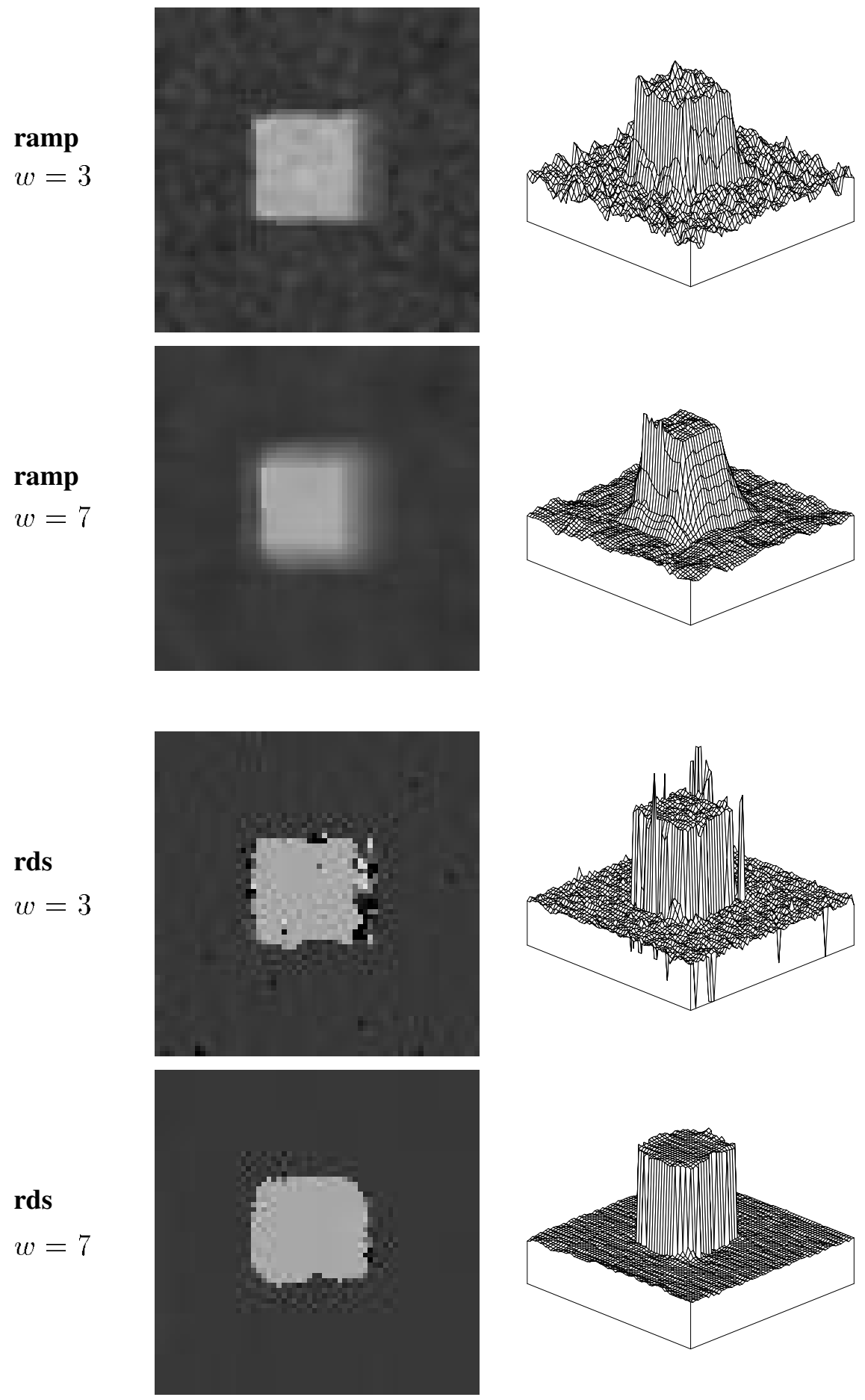

Figure 4: Performance of the SSD algorithm using square windows with sizes $w=3$ and $w=7$ on the ramp and $r d s$ image pairs. 


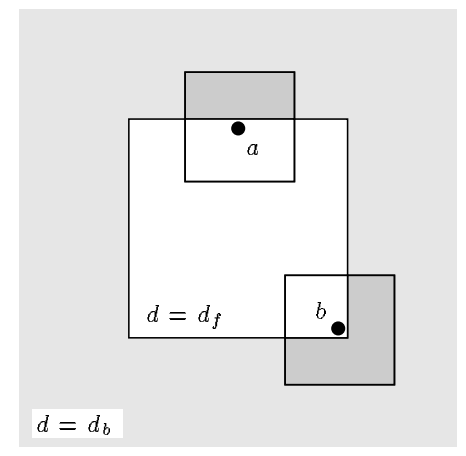

Figure 5: Support for the two disparities $d_{f}$ and $d_{b}$ of foreground and background for two points $a$ and $b$ close to the boundary of the central square.

\subsection{The need for adaptive support regions}

Let us briefly discuss the reasons for boundary blurring by considering the support for two points $a$ and $b$ inside the central square, but close to its boundary (see Figure 5). Both points receive partial support for the two disparities $d_{f}$ and $d_{b}$ of foreground and background respectively, and little support for other disparities. Point $a$, lying next to one of the sides of the square, receives slightly more support from the inside of the square, and is thus correctly found to be at disparity $d_{f}$. Point $b$, lying in the corner, however, receives more support for $d_{b}$, since almost $3 / 4$ of its support region cover the background, and thus is erroneously found to be at disparity $d_{b}$. The overall effect is that corners get rounded since points close to corners are "co-opted" into the wrong disparity. Straight object boundaries are not affected. Note also that no smoothing of the disparity values takes place.

Since the blurring of outlines is caused by support regions that span object boundaries, a possible solution to the problem is to use non-uniform and adaptive support regions. Kanade and Okutomi [Kanade and Okutomi, 1994] have proposed adaptive windows, square windows that extend by different amounts in each of four directions. The optimal window size is found by a greedy algorithm (gradient descent) based on an estimate of disparity uncertainty in the current window. In this paper we propose a different approach: aggregating support with a non-uniform diffusion process.

\section{Aggregating support by diffusion}

Instead of using a fixed window, support can also be aggregated with a weighted support function such as a Gaussian. A convolution with a Gaussian can be implemented using local iterative diffusion [Szeliski and Hinton, 1985] defined by the equation

$$
\frac{\partial E}{\partial t}=\nabla^{2} E
$$


In a discrete system, this yields the update rule

$$
E(i, j, d) \leftarrow(1-4 \lambda) E(i, j, d)+\lambda \sum_{(k, l) \in \mathcal{N}_{4}} E(i+k, j+l, d)
$$

where $\mathcal{N}_{4}=\{(-1,0),(1,0),(0,-1),(0,1)\}$ is the local neighborhood containing the four direct neighbors, and $\lambda$ controls the speed of the diffusion. A value of $\lambda<0.25$ is needed to ensure convergence; we use $\lambda=0.15$ for the experiments reported in this paper.

Aggregation using a finite number of simple diffusion steps yields results that are fairly similar to using square windows. Advantages include the rotational symmetry of the support kernel and the fact that points further away have gradually less influence. The problem of co-opting corners still exists, however.

\subsection{Membrane model}

A problem with simple diffusion is that the size of the support region increases with the number of iterations. In other words, while the diffusion would eventually converge to a uniform support covering the whole image, we are interested in an intermediate time step in which the diffusion has only progressed to a certain amount. We can change this behavior by adding a term to the diffusion equation that measures the amount each current value has diverged from its original value, yielding the membrane equation [Terzopoulos, 1986; Szeliski and Hinton, 1985].

$$
\frac{\partial E}{\partial t}=\nabla^{2} E+\beta\left(E_{0}-E\right)
$$

In the discrete implementation we use

$$
E(i, j, d) \leftarrow[1-\lambda(\beta+4)] E(i, j, d)+\lambda\left[\beta E_{0}(i, j, d)+\sum_{(k, l) \in \mathcal{N}_{4}} E(i+k, j+l, d)\right] .
$$

Unless noted otherwise, we use the parameters $\lambda=0.15$ and $\beta=0.5$ in the experimental results shown in this paper. The $\beta$-term ensures that the diffusion converges to a stable solution not too far from the original values. A closed-from solution for the support function can easily be derived using Fourier analysis (Appendix A).

Figure 6 shows the results of applying our diffusion process to the $r d s$ image pair. The amount of support at each discrete disparity level is shown before diffusion $\left(E_{0}\right)$, after one iteration, and after 10 iterations. Light regions correspond to little support, dark regions indicate strong support. Figure 7 shows the results for accumulating support using the membrane model for the ramp and $r d s$ pairs. The number of diffusion iterations is $n=10$ (the results are almost identical at $n=5$ ).

Using the membrane model alleviates the contour blurring problem to some extent, since the $\beta$-term "ties" the center of each support region to its original value. For very noisy images, however, $\beta$ needs to be chosen quite small to enable enough smoothing for stable matching, making the process more similar to regular diffusion. 


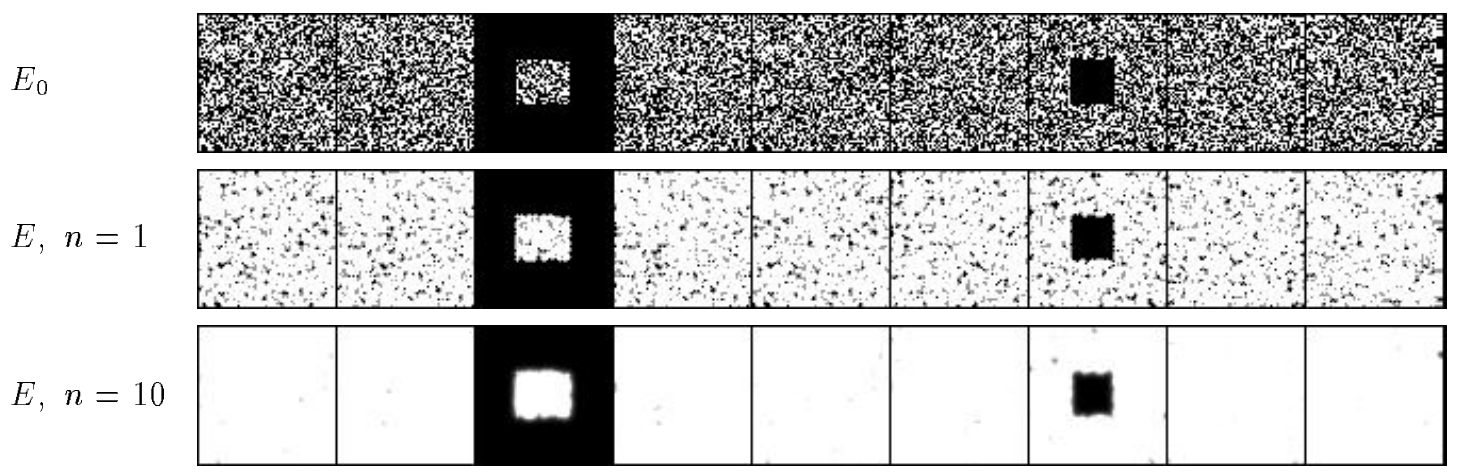

Figure 6: Sections through the disparity space of the $r d s$ image pair during diffusion using the membrane model. The initial disparity space $E_{0}$ is displayed at the top. The diffused disparity space $E$ is shown after one iteration (middle) and after 10 iterations (bottom). Light regions correspond to little support, dark regions indicate strong support.
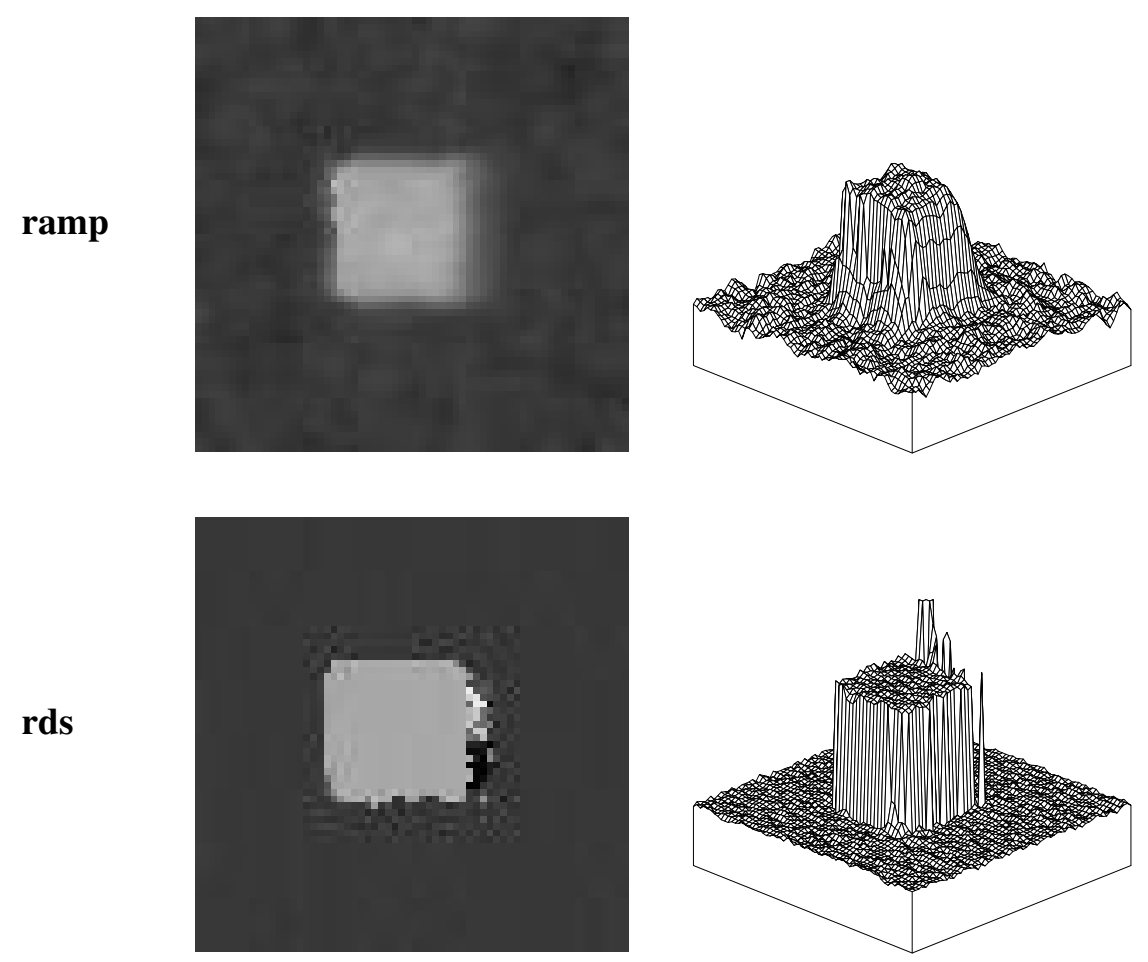

Figure 7: Performance of the membrane model on the ramp and $r d s$ image pairs (gray level images and isometric plots). 


\subsection{Diffusion with local stopping criteria}

A different strategy for preventing both corner co-opting and diffusion to uniformity is to locally stop the diffusion process depending on the distribution of values in each disparity column. To do this, we associate a measure of certainty $C(i, j)$ with each location. Intuitively, this measure should reflect how "clear" a minimum there is among the values $E(i, j, d)$ for all $d$. Given such a measure $C$, we can aggregate support using non-uniform diffusion:

For each $(i, j)$, compute certainties $C$ and $C^{\prime}$ before and after a single iteration of diffusion. If $C>C^{\prime}$, do not diffuse, i.e., restore the old values $E(i, j, d)$ for all $d$.

The idea is that diffusion takes place only at locations of ambiguous matches. Also, certainties never decrease, thus guarantying convergence.

We have experimented with several different certainty measures. In this paper we will discuss two measures, the winner margin, and the entropy. The winner margin $C_{m}$ is the normalized difference between the minimum and the second minimum in a disparity column:

$$
C_{m}(i, j)=\frac{E_{\min 2}-E_{\min }}{\sum_{d} E(i, j, d)} \text {, with } E_{\min }=\min _{d} E(i, j, d), E_{\min 2}=\min _{d, E(i, j, d) \neq E_{\min }} E(i, j, d) .
$$

The second measure $C_{e}$ is the negative entropy of the probability distribution in the disparity column. We convert to probabilities by taking the inverse exponent and normalizing:

$$
C_{e}(i, j)=-\sum_{d} p(d) \log p(d), \text { with } p(d)=\frac{e^{-E(i, j, d)}}{\sum_{d^{\prime}} e^{-E\left(i, j, d^{\prime}\right)}} .
$$

We will develop the idea of converting to probabilities further in the next section.

Figure 8 shows disparity maps for the ramp pair computed with four kinds of diffusion and increasing iterations. The first row shows regular diffusion, the second and third row show diffusion with local stopping based on $C_{m}$ and $C_{e}$. The fourth row shows diffusion using the membrane model for comparison. It is clearly visible that regular diffusion keeps blurring the features as the number of iteration increases, while the other three diffusion processes converge quickly to a stable solution. Which of the three performs best is hard to tell by looking at the disparity maps. In Section 6 we analyze their respective performance based on errors in the computed disparities.

\section{A Bayesian model of stereo matching}

In this section, we develop a Bayesian model for stereo matching that includes both a measurement model corresponding to the matching criterion and a prior Markov Random Field model corresponding to the aggregation function. Our model uses robust (non-Gaussian) statistics to handle gross errors and discontinuities in the surface. We also develop a novel 


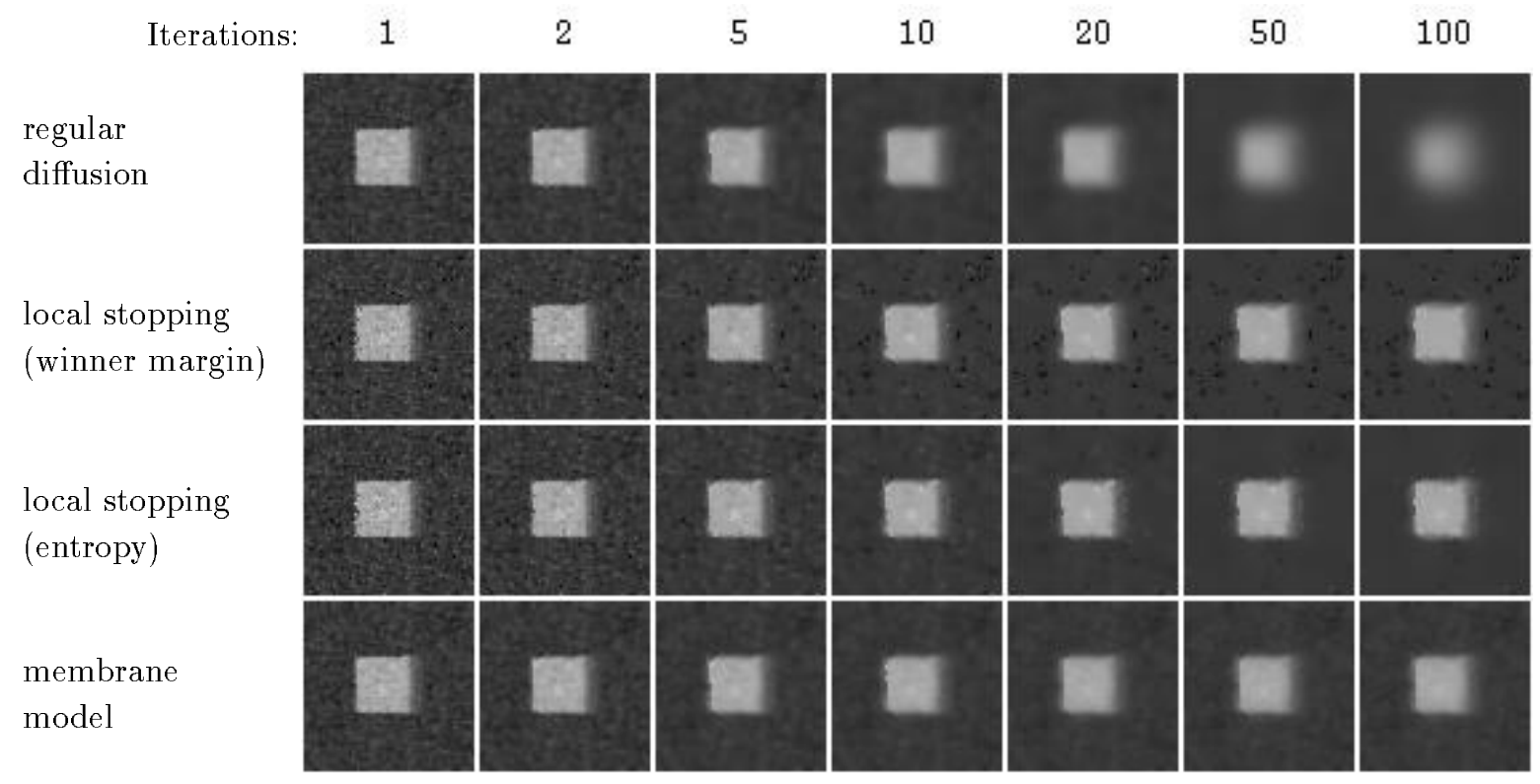

Figure 8: Disparities of the ramp image pair based on diffusion with local stopping compared to regular diffusion and the membrane model.

approximation algorithm that results in a non-linear diffusion process, and show how this produces better results than standard diffusion.

As before, stereo reconstruction is specified as the estimation of a discrete disparity field $d_{i, j}=d\left(x_{i}, y_{j}\right)$ given two (or more) input images $I_{L}(x, y)$ and $I_{R}(x, y)$. Using a Bayesian framework, we first specify a model of image formation, and then derive estimation algorithms from this model.

\subsection{The prior model}

The Bayesian model of stereo image formation consists of two parts. The first part, a prior model for the disparity surface, uses a traditional Markov Random Field (MRF) to encode preferences for smooth surfaces [Geman and Geman, 1984]. This model is specified as a Gibbs distribution $p_{P}$, the exponential of a potential function $E_{P}$ :

$$
p_{P}(\mathbf{d})=\frac{1}{Z_{P}} \exp \left(-E_{P}(\mathbf{d})\right)
$$

where $\mathbf{d}$ is the vector of all disparities $d_{i, j}$ and $Z_{P}$ is a normalizing factor. The potential function itself is the sum of clique potentials

$$
E_{P}(\mathbf{d})=\sum_{c \in C} E_{c}(\mathbf{d})
$$




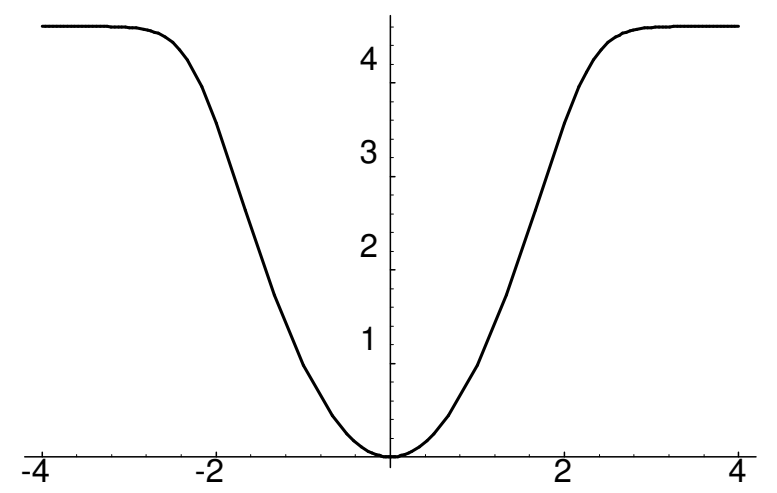

Figure 9: Shape of the robust penalty function $\rho_{P}$ for $\epsilon=0.01$ and $\sigma=1$

which only involve neighboring sites in the field. In this paper, we study only first order fields, where

$$
E_{P}(\mathbf{d})=\sum_{i, j} \rho_{P}\left(d_{i+1, j}-d_{i, j}\right)+\rho_{P}\left(d_{i, j+1}-d_{i, j}\right)
$$

(see [Terzopoulos, 1986; Szeliski, 1989] for generalizations to higher order fields).

When $\rho(x)$ is a quadratic, $\rho(x)=x^{2}$, the field is a Gauss-MRF, and corresponds in a probabilistic sense to a first order regularized (membrane) surface model [Terzopoulos, 1986; Szeliski, 1989]. When $\rho(x)$ is a unit impulse, $\rho(x)=1-\delta(x)$, it corresponds to a MRF that favors fronto-parallel surfaces [Geman and Geman, 1984; Marroquin et al., 1987]. In between these two extremes are functions derived from robust statistics [Huber, 1981], which behave much like surface models with discontinuities [Blake and Zisserman, 1987; Geiger and Girosi, 1991; Black and Rangarajan, 1994]. A wide variety of robust penalty functions are possible [Huber, 1981; Black and Rangarajan, 1994]. In this paper, we use a contaminated Gaussian model,

$$
\rho_{P}(x)=-\log \left(\left(1-\epsilon_{P}\right) \exp \left(-x^{2} / 2 \sigma_{P}^{2}\right)+\epsilon_{P}\right) .
$$

Figure 9 shows the shape of this function for $\epsilon=0.01$ and $\sigma=1$.

\subsection{The measurement model}

The second part of our Bayesian model is the data or measurement model which accounts for differences in intensities between left and right images. This model assumes independent, identically distributed measurement errors,

$$
p_{M}\left(I_{L}, I_{R} \mid \mathbf{d}\right)=\prod_{i, j} p_{M}\left(I_{L}\left(x_{i}+d_{i, j}, y_{j}\right)-I_{R}\left(x_{i}, y_{j}\right)\right)
$$

As mentioned before, traditional stereo matching methods use either a squared intensity error metric (Gaussian noise), $\rho_{M}(x)=\log p_{M}(x)=x^{2}$, or an exact binary matching criterion (e.g., 
for random-dot stereograms or binary features such as edges or the sign of the Laplacian), $\rho_{M}(x)=1-\delta(x)$. In this paper, we again use a contaminated Gaussian model,

$$
\rho_{M}(x)=-\log \left(\left(1-\epsilon_{M}\right) \exp \left(-x^{2} / 2 \sigma_{M}^{2}\right)+\epsilon_{M}\right)
$$

to model both Gaussian noise and possible outliers due to occlusions or non-modeled photometric effects such as specularities.

The posterior distribution, $p\left(\mathbf{d} \mid I_{L}, I_{R}\right)$ can be derived from the prior and measurement models using Bayes' rule,

$$
p\left(\mathbf{d} \mid I_{L}, I_{R}\right) \propto p_{P}(\mathbf{d}) p_{M}\left(I_{L}, I_{R} \mid \mathbf{d}\right)
$$

As is often the case, it is more convenient to study the negative log probability distribution

$$
\begin{aligned}
E(\mathbf{d}) & =-\log p\left(\mathbf{d} \mid I_{L}, I_{R}\right) \\
& =\sum_{i, j} \rho_{P}\left(d_{i+1, j}-d_{i, j}\right)+\rho_{P}\left(d_{i, j+1}-d_{i, j}\right)+\sum_{i, j} \rho_{M}\left(I_{L}\left(x_{i}+d_{i, j}, y_{j}\right)-I_{R}\left(x_{i}, y_{j}\right)\right) .
\end{aligned}
$$

While $p\left(\mathbf{d} \mid I_{L}, I_{R}\right)$ specifies a complete distribution, usually only a single optimal estimate of $d(x, y)$ is desired (but see [Szeliski, 1989] why modeling of uncertainties may be useful). The most commonly studied estimate is the peak of the distribution, or Maximum A Posteriori (MAP) estimate, which is equivalent to minimizing the energy given in (16). Alternative estimates include quantities such as the mean of the distribution [Marroquin et al., 1987].

A variety of techniques have been developed for minimizing (16). Two of the most popular are the Gibbs Sampler [Geman and Geman, 1984; Marroquin et al., 1987] and mean field theory [Geiger and Girosi, 1991]. The Gibbs Sampler randomly chooses values for each $d_{i, j}$ site according to the local distribution determined by the current guesses for a site's neighbors [Geman and Geman, 1984; Szeliski and Hinton, 1985; Barnard, 1989]. This process will in theory converge to a statistically optimal sample, given enough time. Mean field theory updates an estimate of the mean value of $d_{i, j}$ at each site using a deterministic update rule derived from the original probability distribution [Geman and Geman, 1984]. It is not guaranteed to find an optimal estimate, but in practice it often finds a good solution, similar to one available through continuation methods [Blake and Zisserman, 1987].

\subsection{Explicit local distribution model}

The Gibbs Sampler and its variants can produce good solutions, but at the cost of long computation times. Mean field techniques, on the other hand, are not very good at modeling ambiguous estimates, such as multiple potential matches at each pixel. Instead of using either of these two traditional approaches, we will develop a novel estimation algorithm based on modeling the probability distribution of $d_{i, j}$ at each site. To do this, we associate a scalar value between 0 and 1 with each possible discrete value of $d$ at each pixel $(i, j)$, and require that

$$
\sum_{d} p(i, j, d)=1
$$


Our representation is therefore the same as that used by diffusion-based algorithms, i.e., we explicitly model all possible disparities at each pixel, rather than modeling a single estimated disparity as in traditional Gibbs Sampler or mean-field approaches [Barnard, 1989].

To initialize our algorithm, we calculate the probability distribution for each pixel $(i, j)$ based on the intensity errors between matching pixels, i.e.,

$$
p_{0}(i, j, d) \propto \exp \left(-E_{0}(i, j, d)\right)
$$

where

$$
E_{0}(i, j, d)=\rho_{M}\left(I_{L}\left(x_{i}+d, y_{j}\right)-I_{R}\left(x_{i}, y_{j}\right)\right)
$$

is the matching cost of pixel $(i, j)$ at disparity $d$.

To derive the update formula, we start with a basic observation about Markov Random Fields: if the joint probability distribution of all interacting neighbors is known, the local probability distribution of a site is completely determined. To compute this distribution, we take the part of the potential energy (16) which involves $(i, j)$, i.e.,

$$
\tilde{E}\left(d_{i, j} \mid\left\{d_{i+k, j+l}\right\}\right)=E_{0}(i, j, d)+\sum_{(k, l) \in \mathcal{N}_{4}} \rho_{P}\left(d_{i+k, j+l}-d_{i, j}\right),
$$

and turn this into a probability distribution

$$
\tilde{p}\left(d_{i, j} \mid\left\{d_{i+k, j+l}\right\}\right)=p_{0}(i, j, d) \prod_{(k, l) \in \mathcal{N}_{4}} \exp \left(-\rho_{P}\left(d_{i+k, j+l}-d_{i, j}\right)\right) .
$$

We then integrate out all of the neighboring disparities according to their joint probability distribution

$$
p\left(d_{i, j}\right) \propto \sum_{\left\{d_{i+k, j+l}\right\}} \tilde{p}\left(d_{i, j} \mid\left\{d_{i+k, j+l}\right\}\right) p\left(\left\{d_{i+k, j+l}\right\}\right) .
$$

In practice, however, it is impossible to estimate the full joint probability distribution of the neighbors, without resorting to a statistical technique such as the Gibbs Sampler. ${ }^{1}$ Instead, we assume (sub-optimally) that the neighboring disparity columns have independent distributions

$$
p\left(\left\{d_{i+k, j+l}\right\}\right)=\prod_{(k, l) \in \mathcal{N}_{4}} p\left(d_{i+k, j+l}\right)
$$

where the $p\left(d_{i+k, j+l}\right)$ are the current probability density estimates for each neighboring site $(i+k, j+l)$.

The complete update formula is therefore

$$
p\left(d_{i, j}\right) \propto p_{0}(i, j, d) \prod_{(k, l) \in \mathcal{N}_{4}}\left[\sum_{d_{i+k, j+l}^{\prime}} \exp \left(-\rho_{P}\left(d_{i+k, j+l}^{\prime}-d_{i, j}\right)\right) p\left(d_{i+k, j+l}^{\prime}\right)\right]
$$

\footnotetext{
${ }^{1}$ This is not true, however, of 1-D processes such as Markov Random Walks.
} 
or

$$
E(i, j, d) \leftarrow E_{0}(i, j, d)+\sum_{(k, l) \in \mathcal{N}_{4}} \log \left[-\sum_{d^{\prime}} \exp \left(-\rho_{P}\left(d^{\prime}-d\right)-E\left(i+k, j+l, d^{\prime}\right)\right)\right]
$$

For notational and computational convenience, we will introduce a few more additional quantities. The smoothed probability distribution

$$
p_{S}(i, j, d)=\sum_{d^{\prime}} e^{-\rho_{P}\left(d^{\prime}-d\right)} p\left(i, j, d^{\prime}\right)=\sum_{d^{\prime}} w_{P}\left(d^{\prime}-d\right) p\left(i, j, d^{\prime}\right)
$$

is simply the current probability distribution $p(i, j, d)$ after it has been convolved vertically (in disparity) with the smoothing kernel $w_{P}(d) \propto e^{-\rho_{P}(d)}$, with $\sum_{d} w_{P}(d)=1$. It has a corresponding smoothed energy

$$
E_{S}(i, j, d)=-\log p_{S}(i, j, d)
$$

Finally, the update rule can be written as a pair of equations

$$
\begin{aligned}
E(i, j, d) & \leftarrow E_{0}(i, j, d)+\sum_{(k, l) \in \mathcal{N}_{4}} E_{S}(i+k, j+l, d), \\
p(i, j, d) & \leftarrow \frac{e^{-E(i, j, d)}}{\sum_{d^{\prime}} e^{-E\left(i, j, d^{\prime}\right)}} .
\end{aligned}
$$

In practice, since the values of $E(i, j, d)$ are being updated simultaneously at all pixels and disparity, we use a modified version of (28),

$$
E(i, j, d) \leftarrow E_{0}(i, j, d)+\mu\left[E_{S}(i, j, d)+\sum_{(k, l) \in \mathcal{N}_{4}} E_{S}(i+k, j+l, d)\right],
$$

i.e., we weight the neighboring values somewhat less (we use $\mu=0.5$ ) and include the current estimated energy in the update rule.

If we interpret the above equations as a four-step algorithm for iteratively computing the best stereo matches, we see that they are a special instance of a non-linear diffusion process. The smoothing step in (26-27) blurs the current disparity probabilities vertically along a column, thereby enabling different nearby disparities to support each other (depending on the size of $\left.\sigma_{P}\right)$. It also adds a small amount to each probability $\left(\epsilon_{P}\right)$, which in effect limits the largest possible value that $E_{S}$ can take and thus limits the effect of disparity discontinuities.

The update step (30) is identical to a regular diffusion step with $\beta$-terms (membrane model). However, the probability re-normalization step ensures that the energies represent meaningful $\log$ probabilities (in practice, it forces the smallest $E$ to be slightly above 0 ). The robust form of the $E_{0}$ function also ensures that bad matches have only limited effects, thus allowing for occlusions or other non-modeled errors to occur.

For the above algorithm to work well, the various parameters $\left\{\sigma_{P}, \epsilon_{P}, \sigma_{M}, \epsilon_{M}\right\}$ must be set to appropriate values. $\sigma_{M}$ and $\epsilon_{M}$ are based on the expected noise in the image sensor, 

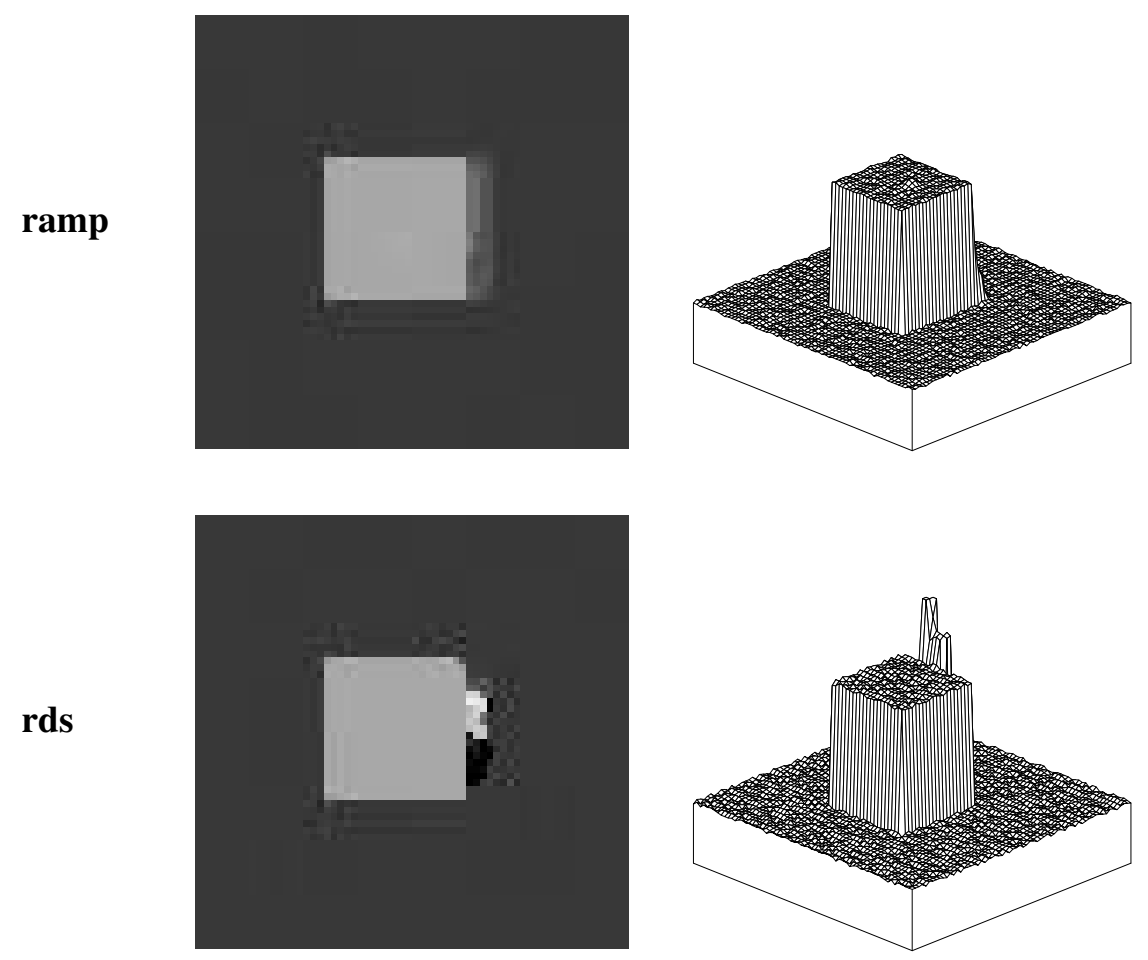

Figure 10: Performance of the probabilistic model on the ramp and $r d s$ image pairs (gray level images and isometric plots).

i.e., $\sigma_{M}$ should be proportional to the regular image noise, while $\epsilon_{M}$ should be the probability of gross errors or occlusions (say $1-10 \%$ ). The choice of $\sigma_{P}$ depends on the class of disparity surfaces which may be expected, i.e., a small $\sigma_{P}$ favors fronto-parallel surfaces. For the experiments presented in this paper, we set $\sigma_{P}=0.1$ and $\epsilon_{P}=0.01$.

Figure 10 shows the results of our probabilistic aggregation technique applied to the ramp and $r d s$ images. We use a different $\sigma_{M}$ for the two image pairs: $\sigma_{M}=2$ for ramp; $\sigma_{M}=20$ for $r d s$, to compensate for the different signal strengths of the two pairs. The other parameters are the same for both image pairs: $\epsilon_{M}=0.1, \sigma_{P}=0.1, \epsilon_{P}=0.01$. The number of diffusion iterations is $n=10$.

\section{Experimental results}

In this section we numerically evaluate the performance of the different algorithms on synthetic images. We also show results for real image data.

For our experiments we use five synthetic image pairs, based on combining three different intensity patterns ramp, rds, and real, and two different disparity patterns, square and bars. We have already introduced the square disparity pattern (Figure 2), and the combinations ramp/square and rds/square (Figure 3). 


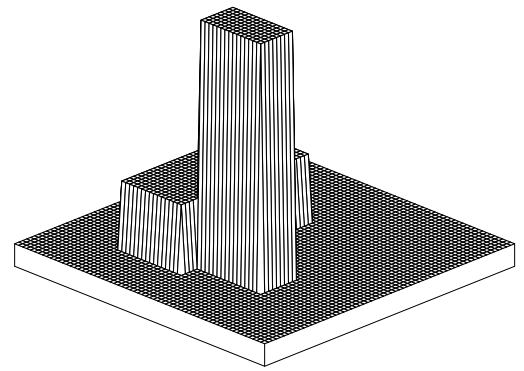

(a)

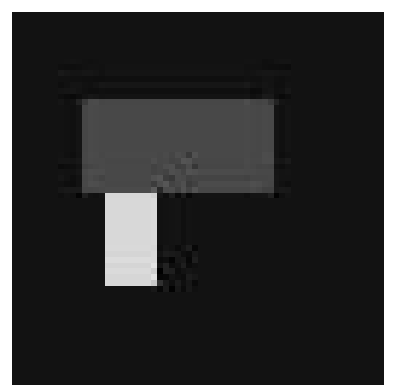

(b)

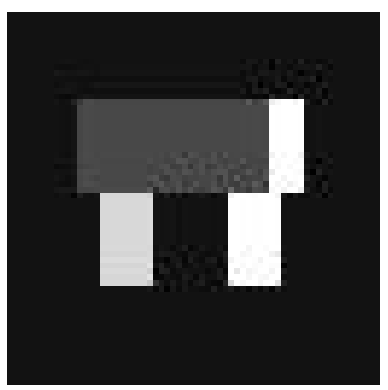

(c)

Figure 11: The bars disparity pattern, containing an ordering constraint violation: (a) isometric plot; (b) gray-level encoding; (c) gray-level encoding with occlusion information.

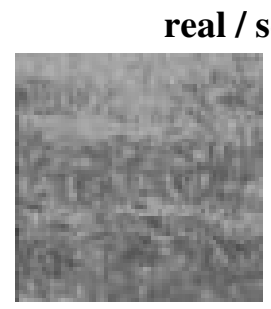

L

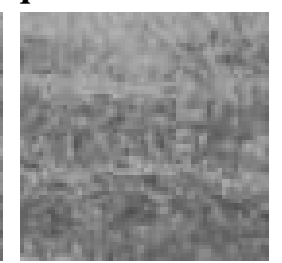

$\mathrm{R}$

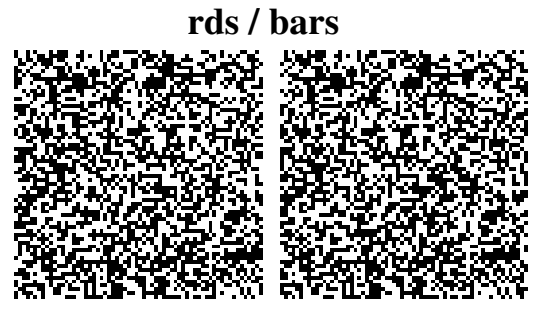

$\mathrm{L}$

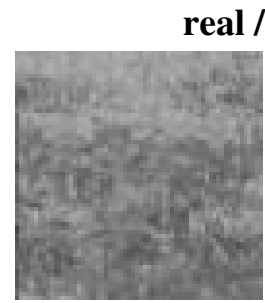

$\mathrm{L}$

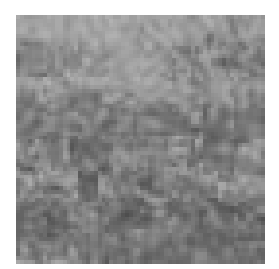

$\mathrm{R}$

Figure 12: The three additional synthetic image pairs.

The new disparity pattern bars consists of two rectangular regions with two different disparities (see Figure 11). The narrow region in the bottom half of the image is displaced by more than twice its width, thus violating the commonly assumed monotonicity (ordering) constraint. Together with the big disparity range, this provides an extra challenge to stereo algorithms, but reflects common situations in real images. The new intensity pattern, real, is part of a real image depicting ground covered with grass.

Figure 12 shows the three new image pairs synthesized using the texture/disparity combinations real/square, rds/bars, and real/bars. We do not use the combination ramp/bars since the narrow region cannot be matched unambiguously, resulting in meaningless disparity error statistics.

We compared the following algorithms: SSD, diffusion using the membrane model, diffusion with local stopping, and diffusion using the probabilistic model. For each algorithm, we varied the parameters: window size (SSD), $\beta, \lambda$ (membrane), certainty measure (local stopping), $\sigma_{M}, \sigma_{P}, \epsilon_{M}, \epsilon_{P}, \mu$ (probabilistic), and the number of iterations (all diffusion algorithms). For each parameter setting, we ran the algorithm on a test set of 40 images (the 5 image pairs with 8 different levels of additive Gaussian noise: $\sigma=0,0.25,0.5,1,2,4,8,16$ ). We tried more than 70 different parameter settings, resulting in about 3000 experiments. In 
each experiment, we compared the computed disparities with the true disparities (ignoring the occluded regions), and collected three different error statistics: mean absolute disparity error, root-mean-square (RMS) disparity error, and the "percentage of bad points", i.e., the percentage of points whose absolute disparity error is greater than $1 / 2$.

Remember that our goal in devising the different algorithms was to recover the occlusion boundaries correctly. The percentage of bad points gives a good indication whether the boundaries are recovered correctly, since this is where the errors are big. For similar reasons, we prefer the RMS error over the mean absolute error since it penalizes outliers more.

First we analyzed the error statistics for each method separately to gain understanding of the effect of the different parameters. Then we chose the best parameters for each method, and compared the different methods with each other. Since space is limited, we only present in detail the results of the second, comparative stage, after briefly discussing the general trends we noticed.

SSD, which we include for comparison, has only one parameter: the size of the support region. The same holds for simple diffusion, where the size of the support region is controlled by the number of iterations. Not surprisingly, the optimal size of the support region depends on the noise level. In general, higher noise levels (or, more precisely, lower signal-to-noise rations) require bigger window sizes. The best window size can also depend on the image.

The membrane model behaves similarly to regular diffusion with a fixed number of iterations. For small noise levels, a value of $\beta$ between $1 / 3$ and 1 usually yields smaller errors than regular diffusion, but not always. Also, as mentioned before, for high noise levels, $\beta$ needs to be chosen quite small to enable enough smoothing for stable matching.

In analyzing regular diffusion with local stopping criteria, we found that the certainty measure is critical. In our experiments, the winner margin $C_{m}$ almost always outperformed the measure based on entropy $C_{e}$. A problem with our definition of local stopping is that an initial wrong but "certain" match can survive. There is clearly a potential for both better certainty measures and different stopping criteria.

The probabilistic model, which performed by far the best, also has the most parameters. We found, however, that many parameters have only small effects and can be set to default values, including $\epsilon_{M}=0.1, \epsilon_{P}=0.01$, and $\mu=0.5$. As expected, a small $\sigma_{P}$ worked best for our test images composed from fronto-parallel surfaces. For real images, we found that $\sigma_{P}$ needs to be chosen slightly higher. The most important parameter is $\sigma_{M}$, which should reflect the strength of the image signal. We used three different values for the three different textures of our test images. Finally, the number of iterations is less critical, since the method seems to converge relatively fast to a stable solution. Higher numbers of iterations are necessary for images containing regions of uniform intensity, such as the real images discussed below.

For direct comparison of the methods, we plot the disparity error versus the noise level on all five image pairs: Figure 13 shows the RMS errors, and Figure 14 shows the percentage of bad points. We compare SSD with a window size of 5 , the membrane model with $\beta=$ 0.5, diffusion with local stopping based on winner margin $C_{m}$, and the probabilistic model with $\epsilon_{P}=0.01, \sigma_{P}=0.1, \epsilon_{M}=0.1$, and $\sigma_{M}=2,8,20$, for ramp, real, and $r d s$ textures respectively. The number of iterations is 10 for all methods. 


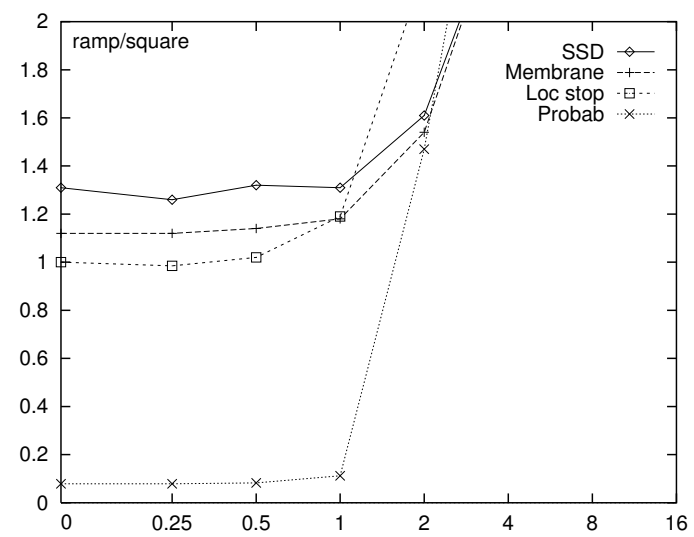

\section{RMS error}
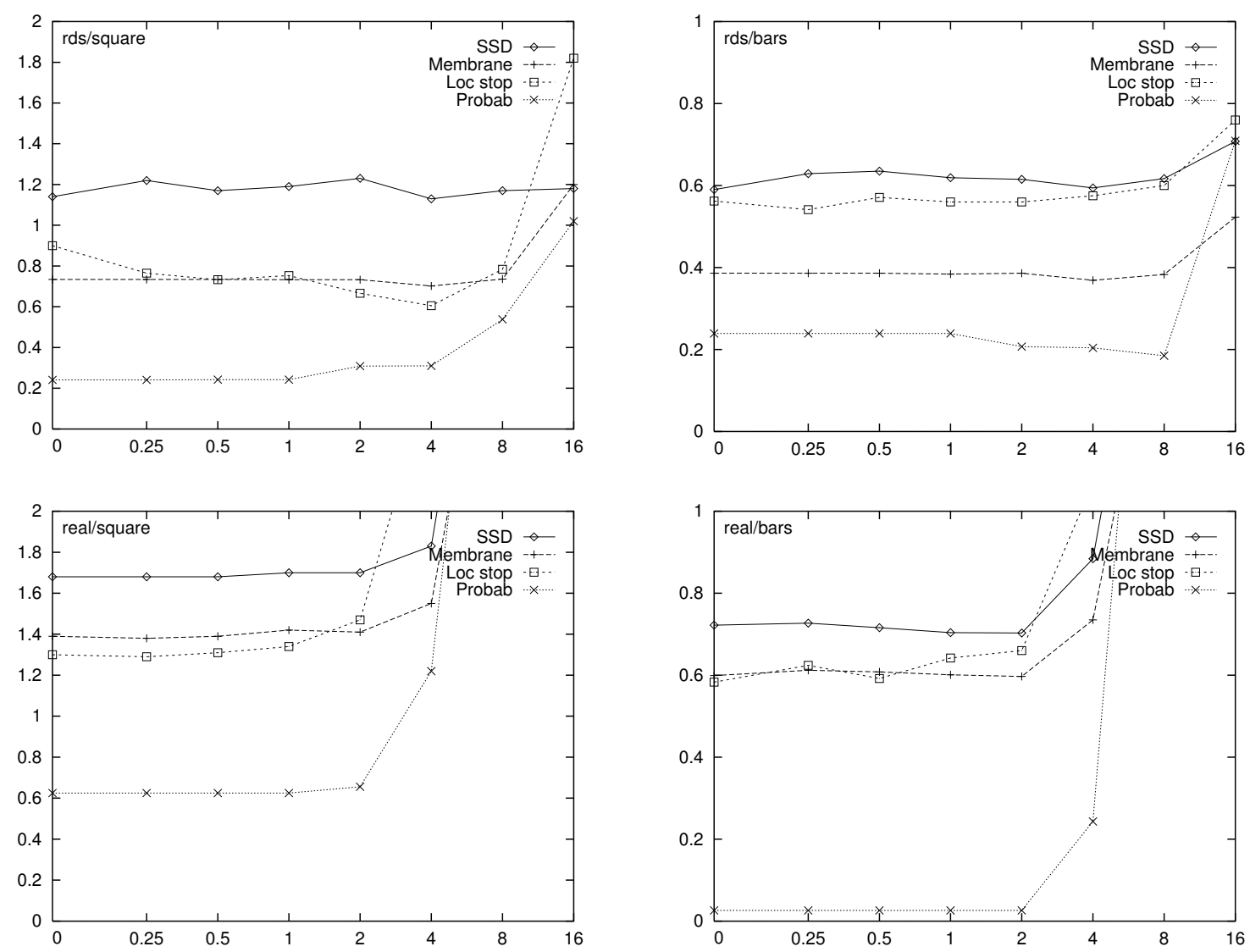

Figure 13: Comparative performance of four stereo algorithms on five test image pairs. The plots show the RMS error of the computed disparities versus the standard deviation of image noise. The error at occluded points is not included. 


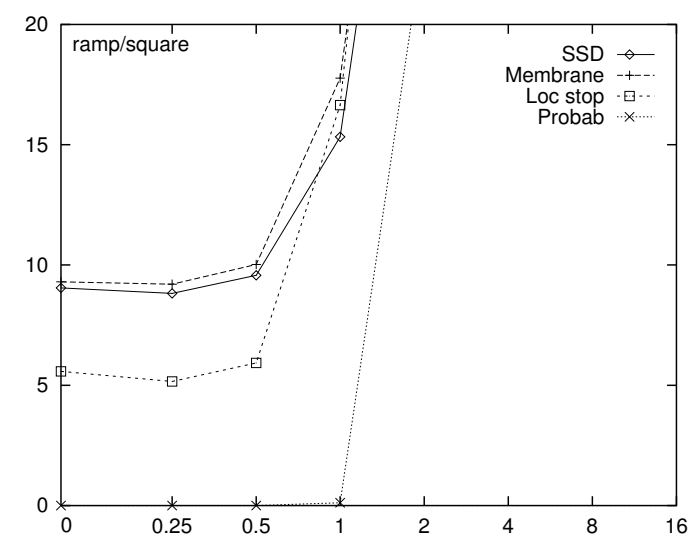

\section{Percentage of bad points}
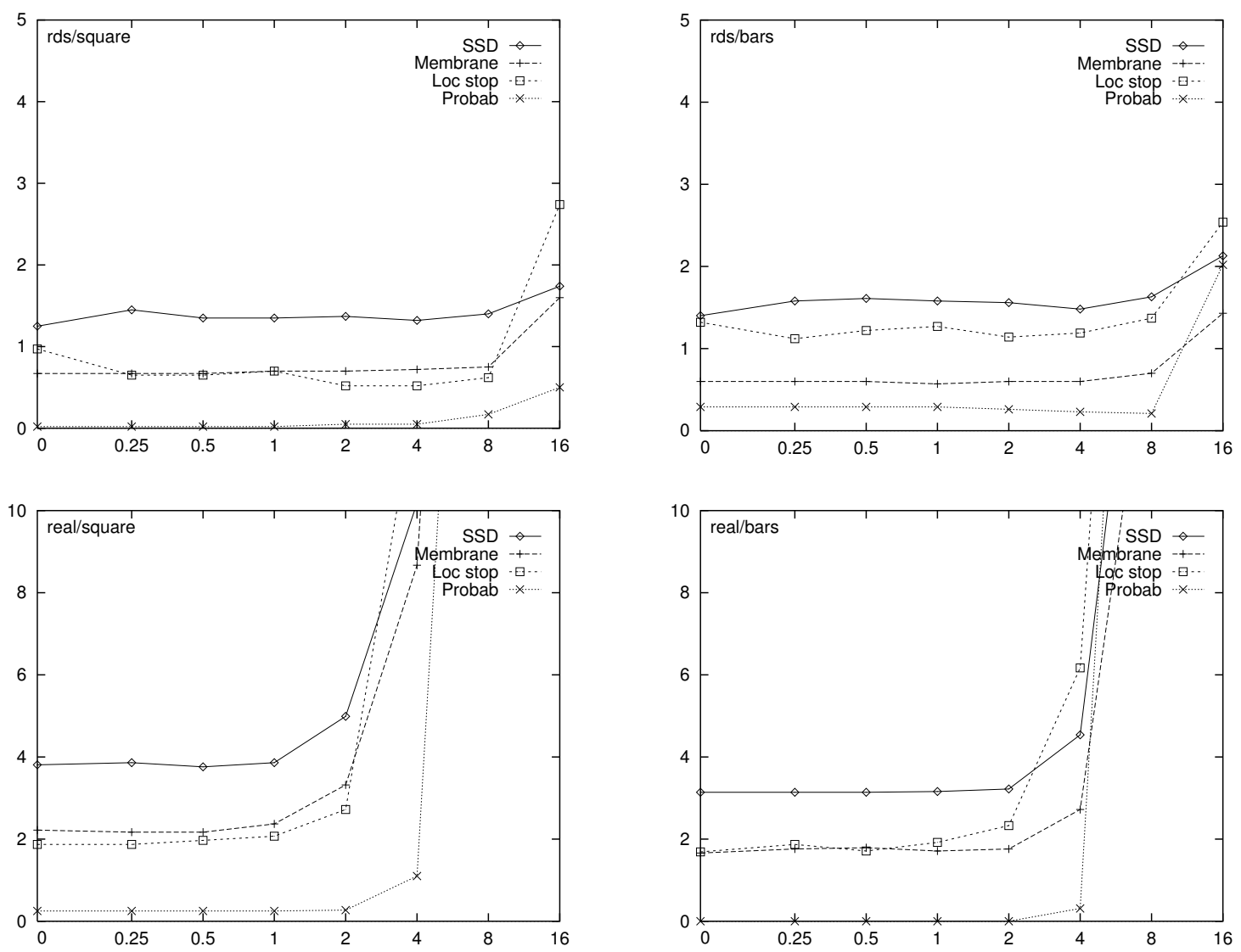

Figure 14: Comparative performance of four stereo algorithms on five test image pairs. The plots show the percentage of points whose absolute disparity error is greater than $1 / 2$, versus the standard deviation of image noise. Occluded points are not considered. 


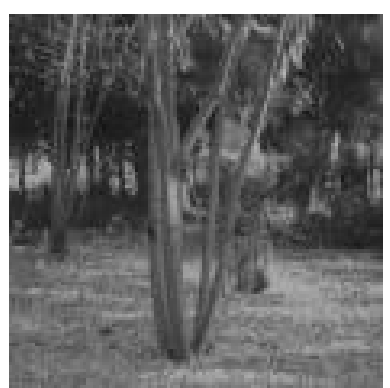

Left

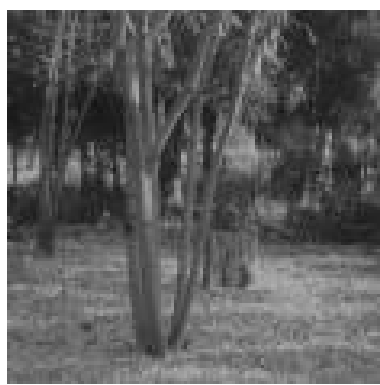

Right

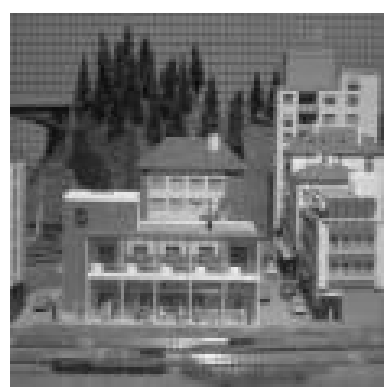

Lower

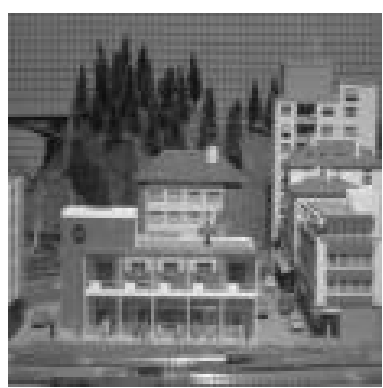

Upper

Figure 15: Tree and town image sets.
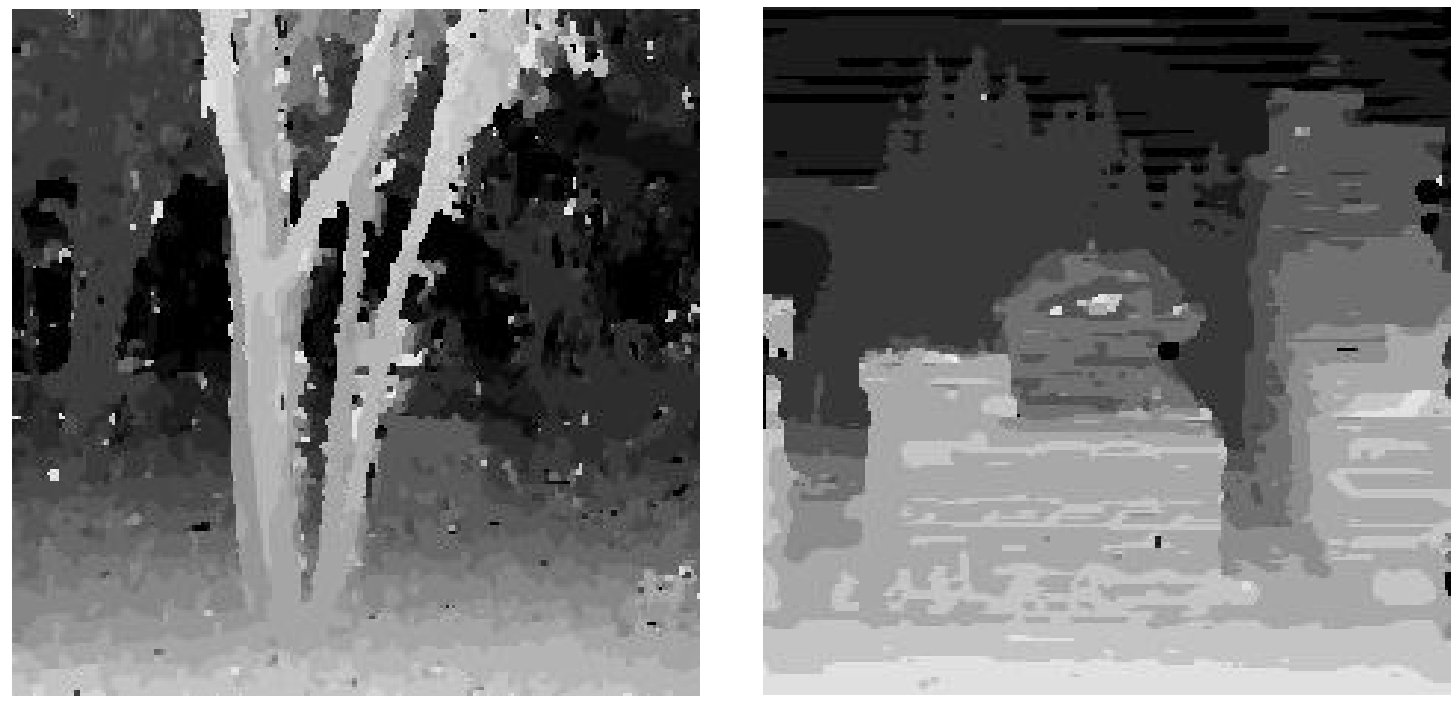

Figure 16: Disparities for tree and town images computed by the probabilistic algorithm.

The probabilistic model clearly beats the three other methods. For small noise levels, the occlusion boundaries are recovered almost perfectly (the percentage of bad points is $0 \%$ in three of five images). Note that the algorithm recovers the "correct" disparity pattern, even though the notion of true disparities is not well defined for ambiguous images such as random dot stereograms.

We also tested our algorithms on real images. We include results of the probabilistic method on images from the SRI's tree sequence and CMU's town sequence (see Figure 15). We used multiple baseline stereo based on five images to initialize the disparity space with the sum of four (appropriately scaled) similarity measures [Okutomi and Kanade, 1993]. Figure 16 shows the disparity maps computed by the probabilistic algorithm after 50 iterations, using the following parameters: $\sigma_{P}=0.4, \epsilon_{P}=0.01, \sigma_{M}=5, \epsilon_{M}=0.1$. Note that we use a bigger $\sigma_{P}$ than before to account for slanted surfaces. 


\section{Discussion}

As we have shown, linear and non-linear diffusion algorithms are an attractive alternative to the adaptive windows introduced by Kanade and Okutomi [Kanade and Okutomi, 1994]. In its simplest form, the membrane algorithm simply requires the iterative summation of neighboring matching costs, with an additional term thrown in to prevent the support region from growing indefinitely. The increased weighting of the central pixel relative to the periphery is sufficient to counteract many of the artifacts introduced by the squared summing window used in SSD. When combined with a local stopping criterion, the resulting non-linear diffusion process has an adaptive support behavior similar to the variable window size algorithm. The inclusion of additional non-linearities in the Bayesian diffusion algorithm improves the performance even more.

In addition to their simplicity and computational efficiency, our non-linear diffusion algorithms can also handle stereograms with more ambiguity than the adaptive window SSD algorithm. Kanade and Okutomi's algorithm is based on locally adjusting the sub-pixel disparity estimate simultaneously with growing the window size. This presupposes that the algorithm is somehow initialized in the vicinity of the true disparity. This is achieved in their synthetic image sequences by using small disparities, and in their real sequences by using a multi-frame version of the basic SSD algorithm [Okutomi and Kanade, 1993]. Image pairs with rapidly varying textures and many potential matches such as the random-dot stereograms used in our experiments could not be handled by their current algorithm. Of course, their basic method could potentially be extended to include a standard multiple disparity search component, but the performance of such a hybrid method is as yet unknown.

In its present form, our algorithm computes monocular rather than binocular disparity maps, i.e., the disparity map is associated with the right image. A binocular representation would remove this restriction, enabling the representation of occluded regions in both left and right images. Extending our diffusion algorithms to a binocular representation is relatively straightforward: the concept of neighbors at the same disparity is modified to define equal disparities in the cyclopean representation of depth, i.e., the depth seen by a camera halfway between the original two. Such a representation would also allow us to deal with occlusions more gracefully, allowing occluded pixels to float to the same disparity as other pixels in the background. However, it is unclear how to extend the Bayesian algorithm, since it requires the re-normalization of disparities along each column in disparity space.

In addition to these extensions, we also plan to study better local stopping criteria based on improved certainty measures. We would also like to investigate multi-resolution versions of our diffusion algorithms to help fill in regions which have few features to match. 


\section{Conclusions}

In this paper, we have demonstrated that diffusion-based aggregation of support is a useful alternative to both traditional area-based correlation and to more recent adaptive window size-based techniques. Our algorithms are simple to implement and computationally efficient, and result in better quality estimates, especially near discontinuities in the disparity surface. The addition of local termination conditions to the basic diffusion process results in a behavior similar to that of adaptively sized windows. Furthermore, our novel non-linear diffusion algorithm derived from a Bayesian model of stereo matching results in markedly improved performance. We believe that further study of the basic support and aggregation methods in stereo matching is central to developing algorithms with improved performance over a wide range of imagery.

\section{Acknowledgements}

A portion of this work was performed while Richard Szeliski was employed at Digital Equipment Corporation's Cambridge Research Lab. 


\section{References}

[Arnold, 1983] R. D. Arnold. Automated stereo perception. Technical Report AIM-351, Artificial Intelligence Laboratory, Stanford University, March 1983.

[Baker, 1980] H. H. Baker. Edge based stereo correlation. In L. S. Baumann, editor, Image Understanding Workshop, pages 168-175. Science Applications International Corporation, April 1980.

[Barnard and Fischler, 1982] S. T. Barnard and M. A. Fischler. Computational stereo. ACM Computing Surveys, 14(4):553-572, December 1982.

[Barnard, 1989] S. T. Barnard. Stochastic stereo matching over scale. International Journal of Computer Vision, 3(1):17-32, 1989.

[Belhumeur and Mumford, 1992] P. N. Belhumeur and D. Mumford. A bayesian treatment of the stereo correspondence problem using half-occluded regions. In IEEE Computer Society Conference on Computer Vision and Pattern Recognition (CVPR'92), pages 506512, Champaign-Urbana, IL, 1992. IEEE Computer Society Press.

[Black and Rangarajan, 1994] M. J. Black and A. Rangarajan. The outlier process: Unifying line processes and robust statistics. In IEEE Computer Society Conference on Computer Vision and Pattern Recognition (CVPR'94), pages 15-22, Seattle, WA, June 1994. IEEE Computer Society Press.

[Blake and Zisserman, 1987] A. Blake and A. Zisserman. Visual Reconstruction. MIT Press, Cambridge, MA, 1987.

[Bolles et al., 1987] R. C. Bolles, H. H. Baker, and D. H. Marimont. Epipolar-plane image analysis: An approach to determining structure from motion. International Journal of Computer Vision, 1:7-55, 1987.

[Cox, 1994] I. J. Cox. A maximum likelihood n-camera stereo algorithm. In IEEE Computer Society Conference on Computer Vision and Pattern Recognition (CVPR'94), pages 733739, Seattle, WA, June 1994. IEEE Computer Society Press.

[Dhond and Aggarwal, 1989] U. R. Dhond and J. K. Aggarwal. Structure from stereo-a review. IEEE Transactions on Systems, Man, and Cybernetics, 19(6):1489-1510, November/December 1989 .

[Geiger and Girosi, 1991] D. Geiger and F. Girosi. Mean field theory for surface reconstruction. IEEE Transactions on Pattern Analysis and Machine Intelligence, 13(5):401-412, May 1991.

[Geiger et al., 1992] D. Geiger, B. Ladendorf, and A. Yuille. Occlusions and binocular stereo. In Second European Conference on Computer Vision (ECCV'92), pages 425-433, Santa Margherita Ligure, Italy, May 1992. Springer-Verlag. 
[Geman and Geman, 1984] S. Geman and D. Geman. Stochastic relaxation, Gibbs distribution, and the Bayesian restoration of images. IEEE Transactions on Pattern Analysis and Machine Intelligence, 6(6):721-741, November 1984.

[Grimson, 1985] W. E. L. Grimson. Computational experiments with a feature based stereo algorithm. IEEE Transactions on Pattern Analysis and Machine Intelligence, 7(1):17-34, January 1985.

[Hoff and Ahuja, 1989] W. Hoff and N. Ahuja. Surfaces from stereo: integrating feature matching, disparity estimation, and contour detection. IEEE Transactions on Pattern Analysis and Machine Intelligence, 11(2):121-136, February 1989.

[Huber, 1981] P. J. Huber. Robust Statistics. John Wiley \& Sons, New York, NY, 1981.

[Intille and Bobick, 1994] S. S. Intille and A. F. Bobick. Disparity-space images and large occlusion stereo. In Third European Conference on Computer Vision (ECCV'94), volume B, pages 179-186, Stockholm, Sweden, May 1994. Springer-Verlag.

[Jenkin et al., 1991] M. R. M. Jenkin, A. D. Jepson, and J. K. Tsotsos. Techniques for disparity measurement. CVGIP: Image Understanding, 53(1):14-30, January 1991.

[Jones and Malik, 1992] D. G. Jones and J. Malik. A computational framework for determining stereo correspondence from a set of linear spatial filters. In Second European Conference on Computer Vision (ECCV'92), pages 397-410, Santa Margherita Ligure, Italy, May 1992. Springer-Verlag.

[Kanade and Okutomi, 1994] T. Kanade and M. Okutomi. A stereo matching algorithm with an adaptive window: Theory and experiment. IEEE Transactions on Pattern Analysis and Machine Intelligence, 16(9):920-932, September 1994.

[Kanade, 1994] T. Kanade. Development of a video-rate stereo machine. In Image Understanding Workshop, pages 549-557, Monterey, CA, November 1994. Morgan Kaufmann Publishers.

[Kang et al., 1995] S. B. Kang, J. Webb, L. Zitnick, and T. Kanade. A multibaseline stereo system with active illumination and real-time image acquisition. In Fifth International Conference on Computer Vision (ICCV'95), pages 88-93, MIT, Cambridge, MA, June 1995. IEEE Computer Society Press.

[Lucas and Kanade, 1981] B. D. Lucas and T. Kanade. An iterative image registration technique with an application in stereo vision. In Seventh International Joint Conference on Artificial Intelligence (IJCAI-81), pages 674-679, Vancouver, 1981.

[Marr and Poggio, 1976] D. Marr and T. Poggio. Cooperative computation of stereo disparity. Science, 194:283-287, October 1976. 
[Marr and Poggio, 1979] D. C. Marr and T. Poggio. A computational theory of human stereo vision. Proceedings of the Royal Society of London, B 204:301-328, 1979.

[Marroquin et al., 1987] J. Marroquin, S. Mitter, and T. Poggio. Probabilistic solution of illposed problems in computational vision. Journal of the American Statistical Association, 82(397):76-89, March 1987.

[Matthies et al., 1989] L. H. Matthies, R. Szeliski, and T. Kanade. Kalman filter-based algorithms for estimating depth from image sequences. International Journal of Computer Vision, 3:209-236, 1989.

[Ohta and Kanade, 1985] Y. Ohta and T. Kanade. Stereo by intra- and inter-scanline search using dynamic programming. IEEE Transactions on Pattern Analysis and Machine Intelligence, $7(2): 139-154$, March 1985.

[Okutomi and Kanade, 1992] M. Okutomi and T. Kanade. A locally adaptive window for signal matching. International Journal of Computer Vision, 7(2):143-162, April 1992.

[Okutomi and Kanade, 1993] M. Okutomi and T. Kanade. A multiple-baseline stereo. IEEE Transactions on Pattern Analysis and Machine Intelligence, 15(4):353-363, 1993.

[Olsen, 1990] S. I. Olsen. Stereo correspondence by surface reconstruction. IEEE Transactions on Pattern Analysis and Machine Intelligence, 12(3):309-314, March 1990.

[Pollard et al., 1985] S. B. Pollard, J. E. W. Mayhew, and J. P. Frisby. PMF: A stereo correspondence algorithm using a disparity gradient limit. Perception, 14:449-470, 1985.

[Prazdny, 1985] K. Prazdny. Detection of binocular disparities. Biological Cybernetics, $52(2): 93-99,1985$.

[Quam, 1984] L. H. Quam. Hierarchical warp stereo. In Image Understanding Workshop, pages 149-155, New Orleans, Louisiana, December 1984. Science Applications International Corporation.

[Ryan et al., 1980] T. W. Ryan, R. T. Gray, and B. R. Hunt. Prediction of correlation errors in stereo-pair images. Optical Engineering, 19(3):312-322, May/June 1980.

[Scharstein, 1994] D. Scharstein. Matching images by comparing their gradient fields. In 12th International Conference on Pattern Recognition (ICPR'94), volume 1, pages 572575, Jerusalem, Israel, October 1994.

[Seitz, 1989] P. Seitz. Using local orientation information as image primitive for robust object recognition. In SPIE Visual Communications and Image Processing IV, volume 1199, pages $1630-1639,1989$. 
[Szeliski and Hinton, 1985] R. Szeliski and G. Hinton. Solving random-dot stereograms using the heat equation. In IEEE Computer Society Conference on Computer Vision and Pattern Recognition (CVPR'85), pages 284-288, San Francisco, CA, June 1985. IEEE Computer Society Press.

[Szeliski, 1989] R. Szeliski. Bayesian Modeling of Uncertainty in Low-Level Vision. Kluwer Academic Publishers, Boston, MA, 1989.

[Terzopoulos, 1986] D. Terzopoulos. Regularization of inverse visual problems involving discontinuities. IEEE Transactions on Pattern Analysis and Machine Intelligence, 8(4):413424, July 1986.

[Tian and Huhns, 1986] Q. Tian and M. N. Huhns. Algorithms for subpixel registration. Computer Vision, Graphics, and Image Processing, 35:220-233, 1986.

[Witkin et al., 1987] A. Witkin, D. Terzopoulos, and M. Kass. Signal matching through scale space. International Journal of Computer Vision, 1:133-144, 1987.

[Yuille and Poggio, 1984] A. L. Yuille and T. Poggio. A generalized ordering constraint for stereo correspondence. A.I. Memo 777, Artificial Intelligence Laboratory, Massachusetts Institute of Technology, Cambridge, MA, May 1984.

[Zabih and Woodfill, 1994] R. Zabih and J. Woodfill. Non-parametric local transforms for computing visual correspondence. In Third European Conference on Computer Vision (ECCV'94), volume II, pages 151-158, Stockholm, Sweden, May 1994. Springer-Verlag. 


\section{A Support function for the membrane model}

The support function (i.e., impulse response or kernel) for the membrane diffusion model is a function which can be convolved with the original input data $E_{0}$ to yield the final value of $E$. This function can be computed by setting $E_{0}$ to a unit impulse $E(i, j)=\delta(i) \delta(j)$, and setting the r.h.s. of (6) to 0 .

For the discrete case (7), this involves solving the coupled set of equations

$$
\beta(\delta(i) \delta(j)-f(i, j))+\sum_{(k, l) \in \mathcal{N}_{4}}(f(i+k, j+l)-f(i, j))=0
$$

(the support function is the same for all disparity levels $d$ ). Re-writing these in the Fourier domain, we obtain

$$
\beta\left(1-F\left(\omega_{x}, \omega_{y}\right)\right)+\sum_{(k, l) \in \mathcal{N}_{4}}\left(F\left(\omega_{x}, \omega_{y}\right) e^{j\left(k \omega_{x}+l \omega_{y}\right)}-F\left(\omega_{x}, \omega_{y}\right)\right)=0
$$

or

$$
F\left(\omega_{x}, \omega_{y}\right)=\frac{\beta}{\beta+4-2 \cos \omega_{x}-2 \cos \omega_{y}} .
$$

While the inverse transform of $F\left(\omega_{x}, \omega_{y}\right)$ has no closed form solution, it is simple enough to compute numerically (see Figure 17 for a plot).
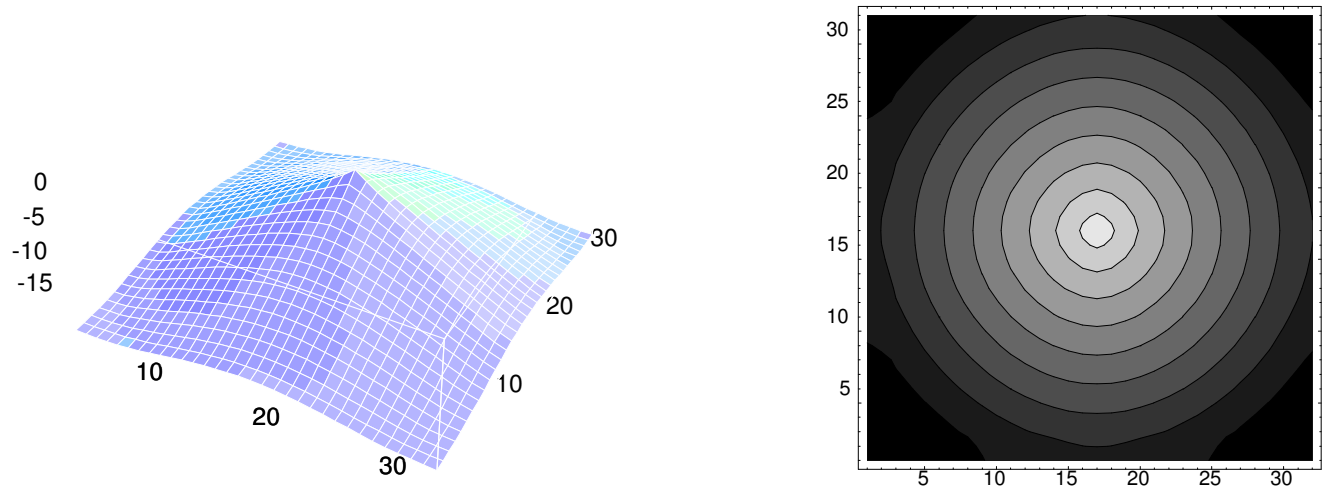

Figure 17: Shape of the membrane support function for $\beta=0.7: 3-\mathrm{D}$ plot and contour plot. 